\title{
Discrete LPV Based Parameter Estimation For T1DM Patients By Using Dual Extended Kalman Filtering Method*
}

\author{
Levente Kovács $^{1}$, Máté Siket ${ }^{1}$, Imre Rudas ${ }^{2}$, Anikó Szakál ${ }^{2}$, György Eigner ${ }^{1}$
}

\begin{abstract}
In case of physiological systems state and parameter estimation is a crucial question. It is key to describe given patient population with appropriate accuracy. Furthermore, state feedback kind of applications also require some sort of estimation procedure in order to get internal information about the controlled system. Linear parameter varying (LPV) framework is beneficial for controller design as well. However, to realize the necessary scheduling parameters, estimation of both state variables and model parameters is needed. A possible solution is the application of Dual Extended Kalman Filter (DEKF) which is able to estimate these signals. The developed framework can be used to design LPV based controller in our further work. In this study we introduce our developed DEKF solution by using the widely applied Cambridge Type 1 Diabetes Mellitus (T1DM) model for virtual patient generation. We have found that our solution is able to estimate the state variables with good accuracy. The variation of parameters can also be tracked by using the proposed solution.
\end{abstract}

\section{INTRODUCTION}

Diabetes mellitus (DM) is a disorder of the natural blood glucose concentration regulatory system. The prevalence of DM is constantly rising, today presumably more than 425 million people are affected worldwide [1]. It is primarily characterized by an elevated fasting blood glucose (BG) level (called hyperglycemia), caused by a relative or absolute deficiency in insulin. The latter one is also called type 1 diabetes mellitus (T1DM), which is the main focus of this work. During an exactly not known autoimmune reaction the insulin producing $\beta$-cells die, thus patients in such a condition are in permanent need of exogenous insulin [2].

On the other hand, during exogenous insulin overdose the BG level can fall under a critical level leading to serious complications, thus it is a twofold task. The BG level has to be kept in a narrow range with low variability, this is the so-called tight glycemic control (TGC). In order to maintain TGC one has to provide BG level feedback with the highest possible sampling frequency. Current continuous glucose monitoring systems (CGMS) achieve 5-minutes-long sampling periods [3]. The sparseness of the measurements is only one obstacle in the control design procedure, others

This project has received funding from the European Research Council (ERC) under the European Union's Horizon 2020 research and innovation programme (grant agreement No 679681).

1 The Authors are with the Physiological Controls Research Center within the Research, Innovation and Service Center of Óbuda University, Budapest, Hungary kovacs@uni-obuda.hu, siket.mate@gmail.com, eigner.gyorgy@nik.uni-obuda.hu

2 The Authors are with the Research, Innovation and Service Center of Óbuda University, Budapest, Hungary rudas@uni-obuda.hu, szakal@uni-obuda.hu include noises and disturbances or the uncertainty of the mathematical model [4]-[6].

The idea of a completely autonomous insulin delivery unit (Artificial Pancreas) is not a novel one, however the final solution is yet to come. Among others the difficulties mentioned above are the main hindrances of the TGC. In order to overcome these issues the filtering of the measurements, the estimation of the internal states and parameters are fundamental. For the estimation and filtering purposes a possible solution can be a DEKF algorithm [7], [8]. Typical T1DM models are non-linear ones, for the practical handling of these systems linear parameter varying technique is a proven method.

The paper is structured as follows. First, we introduce the investigated models in section II and II-C. After the discretization method is introduced in subsection II-D. Afterwards the numerical investigations are presented in section IV. Finally, our conclusions are drawn.

\section{APPLIED MODEL}

\section{A. THE CAMBRIDGE MODEL}

The Cambridge model [9], [10] is a non-linear, complex model aiming to formulate virtual patients with T1DM condition. The model consists of 10 state equations and supplementary equations describing additional physiological phenomena by using non-linear functions. The model equations are the following:

$$
\begin{array}{rrc}
\dot{Q}_{1}(t)= & \frac{D_{2}(t)}{\tau_{D}}-F_{01 c}-F_{R}-x_{1}(t) Q_{1}(t) \\
& +k_{12} Q_{2}(t)+E G P_{0}\left(1-x_{3}(t)\right), \\
\dot{Q}_{2}(t)= & Q_{1}(t) x_{1}(t)-\left(k_{12}+x_{2}(t)\right) Q_{2}(t), \\
\dot{I}(t)= & \frac{S_{2}(t)}{\tau_{S} V_{I}}-k_{e} I(t), \\
\dot{x}_{1}(t)= & -k_{a 1} x_{1}(t)+k_{b 1} I(t), \\
\dot{x}_{2}(t)= & -k_{a 2} x_{2}(t)+k_{b 2} I(t), \\
\dot{x}_{3}(t)= & -k_{a 3} x_{3}(t)+k_{b 3} I(t), \\
\dot{D}_{1}(t)= & \frac{100 A_{g}}{M_{w g}} w(t)-\frac{D_{1}(t)}{\tau_{D}}, \\
\dot{D}_{2}(t)= & \frac{D_{1}(t)}{\tau_{D}}-\frac{D_{2}(t)}{\tau_{D}}, \\
\dot{S}_{1}(t)= & u(t)-\frac{S_{1}(t)}{\tau_{S}}, \\
\dot{S}_{2}(t)= & \frac{S_{1}(t)}{\tau_{S}}-\frac{S_{2}(t)}{\tau_{S}},
\end{array}
$$

where $Q_{1}(t)$ [mmol] is the glucose content in the accessible compartment, $Q_{2}(t)$ [mmol] is the glucose content in the non-accessible compartment, $I(t)[\mathrm{mU} / \mathrm{L}]$ is the insulin concentration in the blood. Indirect effects of insulin on blood 
glucose are expressed in $x_{1}(t), x_{2}(t)$ and $x_{3}(t) . D_{1}(t)$ and $D_{2}(t)$ form the food absorption sub-model. $S_{1}(t)$ and $S_{2}(t)$ form the insulin absorption sub-model.

Additional non-linearities are described by [10]:

$$
\begin{gathered}
F_{01 c}= \begin{cases}F_{01}, & \text { if } G(t) \geq 4.5 \mathrm{mmol} / 1 \\
F_{01} G(t) / 4.5, & \text { otherwise }\end{cases} \\
F_{R}= \begin{cases}0.003 V_{G}(G(t)-9), & \text { if } G(t) \geq 9 \mathrm{mmol} / 1 \\
0, & \text { otherwise }\end{cases} \\
G(t)=\frac{Q_{1}(t)}{V_{G}} \\
V_{G}=0.16 B W \\
V_{I}=0.12 B W,
\end{gathered}
$$

where $G(t)$ is the actual output of the system, namely, the blood glucose concentration [mmol/L].

\section{B. SENSOR NOISE MODEL FOR DATA GENERATION}

In case of real CGMS applications the modeling of sensor noise is a fundamental issue. The BG level is mostly measured in the subcutaneous space of the abdomen or arm which can be different from the average BG level of the blood. Also, there are effects which corrupt the measurement (e.g. time delay) or heat which can lead to sensor decalibration. In this study we have applied the following model [11] to map these phenomena which can be directly connected to the introduced model described by (1)-(16).

$$
\begin{gathered}
e_{k}=0.7\left(e_{k-1}+v_{k}\right), \quad k \geq 1, \\
v_{k} \sim N_{i i d}(0,1), \\
\eta_{k}=\xi+\lambda \sinh \left(\frac{e_{k}-\gamma}{\delta}\right), \\
\dot{G}_{s u b}(t)=\frac{1}{\tau_{s u b}}\left(G(t)-G_{s u b}(t)\right), \\
G_{C G M}(k T)=G_{s u b}(k T)+\eta_{k},
\end{gathered}
$$

where we applied the parameters given by [11]: $\tau_{s u b}=15 \mathrm{~min}$, $\xi=-5.471 \mathrm{mg} / \mathrm{dL}, \lambda=15.96 \mathrm{mg} / \mathrm{dL}, \gamma=-0.5444$ and $\delta=1.6898$. The Brownian motion like term can be initialized with $e_{0} \sim$ $N_{i i d}(0,1)$. The noisy measurement data can be calculated by using the sampled output of the virtual patient system $G(t)$ is used to generate noisy measurement data with $T=5 \mathrm{~min}$ sampling time.

\section{LPV MODELING}

General form of a given LPV state-space (LPV-SS) system can be written in the following way [12]:

$$
\begin{aligned}
& \dot{\mathbf{x}}(t)=\mathbf{A}(\mathbf{p}(\mathbf{t})) \mathbf{x}(t)+\mathbf{B}(\mathbf{p}(\mathbf{t})) \mathbf{u}(t), \\
& \mathbf{y}(t)=\mathbf{C}(\mathbf{p}(\mathbf{t})) \mathbf{x}(t)+\mathbf{D}(\mathbf{p}(\mathbf{t})) \mathbf{u}(t),
\end{aligned}
$$

where the $\mathbf{p}(t)$-dependent state, control input, output, input feed-forward and output feed-forward matrices are $\mathbf{A}(\mathbf{p}(t)) \in \mathbb{R}^{n \times n}, \mathbf{B}(\mathbf{p}(t)) \in \mathbb{R}^{n \times m}, \mathbf{C}(\mathbf{p}(t)) \in \mathbb{R}^{k \times n}$,
$\mathbf{D}(\mathbf{p}(t)) \in \mathbb{R}^{k \times m}$. The $\mathbf{x}(t) \in \mathbb{R}^{n}, \mathbf{y}(t) \in \mathbb{R}^{k}$ and $\mathbf{u}(t) \in$ $\mathbb{R}^{m}$ are the state, output and control input vectors. The LPV modeling parameter is $\mathbf{p}(t)=\left[p_{1}(t) \ldots p_{R}(t)\right]$ parameter vector built up from scheduling parameters $p_{i}(t)$.

In case of the applied Cambridge model the efficient handling of non-linearities (caused by the multiplication of states and by the additional non-linear variables $\left(F_{01 c}, F_{R}\right)$ ) is a crucial issue. One possible way to solve these problems is to enclose these terms into the $\mathbf{p}(t)$ parameter vector, this leads to a quasi-LPV (qLPV) model [13]. However, this model can be designed in several ways, depending on the selection of the parameters. In an earlier study we have examined the effect of different parameter vectors [14]. We came to the conclusion that the main difference is in the applicability of the discretization method described in Section (II-D). By selecting the parameter vector described in (25) the condition: $Q_{1}(t)>0$ has to be satisfied, which is a reasonable presumption considering the physiological property of the state variable.

Based on our preliminary investigations and assumptions we have developed the following state space kind of LPV model by using (1)-(16) and (22)-(23):

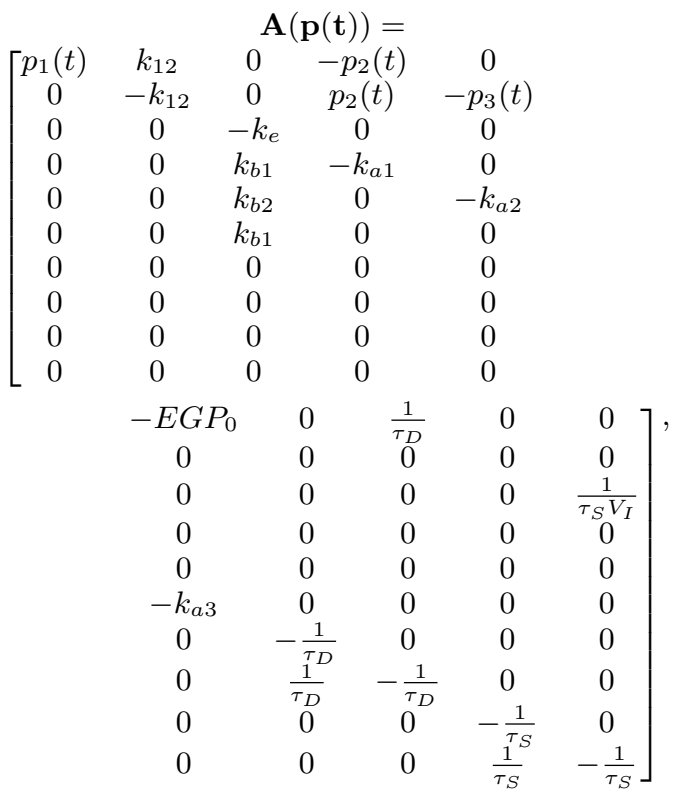

$$
\mathbf{B}=\left[\begin{array}{llllllllll}
0 & 0 & 0 & 0 & 0 & 0 & 0 & 0 & 1 & 0
\end{array}\right]^{\top},
$$

$$
\begin{aligned}
& \mathbf{C}=\left[\begin{array}{llllllllll}
\frac{1}{V_{G}} & 0 & 0 & 0 & 0 & 0 & 0 & 0 & 0 & 0
\end{array}\right] \\
& \mathbf{D}=[0], \\
& \mathbf{p}(t)=\left[p_{1}(t), p_{2}(t), p_{3}(t)\right]= \\
& {\left[\frac{E G P_{0}-F_{01 c}-F_{R}}{Q_{1}(t)}, Q_{1}(t), Q_{2}(t)\right] \text {. }}
\end{aligned}
$$




\section{DISCRETIZATION METHOD}

In our earlier findings [14], we concluded that the Complete LPV-SS discretization is able to provide the best accuracy among other methods [15]. Although this method requires particular attention during the construction of the LPV model, because of possible invertibility issues. The discrete counterparts of the continuous time LPV state space model can be realized as follows, where discrete time system matrices are marked with $d$ indices [15], [16]:

$$
\begin{gathered}
\mathbf{A}_{d}(\mathbf{p}(k))=e^{(\mathbf{A}(\mathbf{p}(k T)) T}, \\
\mathbf{B}_{d}(\mathbf{p}(k))=\mathbf{A}^{-1}\left(\mathbf{p}(k T)\left(e^{(\mathbf{A}(\mathbf{p}(k T)) T)}-\mathbf{I}\right)\right. \\
\mathbf{B}(\mathbf{p}(k T)), \\
\mathbf{C}_{d}(\mathbf{p}(k))=\mathbf{C}(\mathbf{p}(k T)), \\
\mathbf{D}_{d}(\mathbf{p}(k))=\mathbf{D}(\mathbf{p}(k T)),
\end{gathered}
$$

\section{DUAL EXTENDED KALMAN FILTER}

Kalman filter became a widely used mathematical algorithm for filtering and also for state estimation purposes. The algorithm takes into account the statistical uncertainty of the dynamic mathematical model and the sensors [7]. Formerly it was used only on linear system, however soon non-linear approximations became the main focus of the researchers. One of the longest established of them is the Extended Kalman Filter (EKF). The special case when two EKF run concurrently in parallel is called a Dual Extended Kalman Filter (DEKF) [17]-[19]. This method creates the favourable opportunity of parallel state and parameter estimation of the system. Application of DEKF algorithm related to physiological application is beneficial since not only the internal state variables can be predicted but also the patient specific model parameters as well to map differences between patients on the model level. Furthermore, the variation of the patient's own parameters over time can also be tracked in this way. In case of LPV based control these predictions can be used to generate the $\mathbf{p}(t)$ connected to a LPV controller which is able to adapt in this way to the specific needs of given patients on the modeling.

After the initialization of the DEKF the algorithm consists of a two-step procedure in both state and parameter instances as it is introduced as follows [18], [19]:

\section{Initialization:}

- State

$$
\hat{\mathbf{x}}=E\langle\mathbf{x}\rangle, \quad \mathbf{P}_{\mathbf{x}_{0}}=E\left\langle\left(\mathbf{x}_{0}-\hat{\mathbf{x}}_{0}\right)\left(\mathbf{x}_{0}-\hat{\mathbf{x}}_{0}\right)^{\top}\right\rangle,
$$

- Parameter

$$
\hat{\mathbf{w}}=E\langle\mathbf{w}\rangle, \quad \mathbf{P}_{\mathbf{w}_{0}}=E\left\langle\left(\mathbf{w}-\hat{\mathbf{w}}_{0}\right)\left(\mathbf{w}-\hat{\mathbf{w}}_{0}\right)^{\top}\right\rangle,
$$

\section{Predict:}

- State

$$
\begin{gathered}
\hat{\mathbf{x}}_{k}^{-}=\mathbf{F}\left(\hat{\mathbf{x}}_{k-1}, \mathbf{u}_{k}, \hat{\mathbf{w}}_{k}^{-}\right), \\
\mathbf{P}_{\mathbf{x}_{k}}^{-}=\mathbf{A}_{k-1} \mathbf{P}_{\mathbf{x}_{k-1}} \mathbf{A}_{k-1}^{\top}+\mathbf{Q} .
\end{gathered}
$$

- Parameter

$$
\begin{gathered}
\hat{\mathbf{w}}_{k}^{-}=\hat{\mathbf{w}}_{k-1}^{-}, \\
\mathbf{P}_{\mathbf{w}_{k}}^{-}=\lambda^{-1} \mathbf{P}_{\mathbf{w}_{k-1}},
\end{gathered}
$$

where $\mathbf{Q}$ is the process noise covariance matrix, $\lambda$ is the forgetting factor $(0,1]$.

Update:

- State

$$
\begin{gathered}
\mathbf{K}_{k}^{\mathbf{x}}=\mathbf{P}_{\mathbf{x}_{k}}^{-} \mathbf{C}^{\top}\left(\mathbf{C P}_{\mathbf{x}_{k}}^{-} \mathbf{C}^{\top}+\mathbf{R}^{\mathbf{x}}\right)^{-1}, \\
\hat{\mathbf{x}}_{k}=\hat{\mathbf{x}}_{k}^{-}+\mathbf{K}_{k}^{\mathbf{x}}\left(\mathbf{z}_{k}-\mathbf{y}_{k}^{-}\right), \\
\mathbf{P}_{\mathbf{x}_{k}}=\left(\mathbf{I}-\mathbf{K}_{k}^{\mathbf{x}} \mathbf{C}\right) \mathbf{P}_{\mathbf{x}_{k}}^{-},
\end{gathered}
$$

- Parameter

$$
\begin{gathered}
\mathbf{C}_{k}^{\mathbf{w}}=\mathbf{C} \frac{\partial \mathbf{F}(\hat{\mathbf{x}}, \hat{\mathbf{w}})}{\partial \hat{\mathbf{w}}} \\
\mathbf{K}_{k}^{\mathbf{w}}=\mathbf{P}_{\mathbf{w}_{k}}^{-}\left(\mathbf{C}_{k}^{\mathbf{w}}\right)^{\top}\left(\mathbf{C}_{k}^{\mathbf{w}} \mathbf{P}_{\mathbf{w}_{k}}^{-}\left(\mathbf{C}_{k}^{\mathbf{w}}\right)^{\top}+\mathbf{R}^{\mathbf{w}}\right)^{-1} \\
\hat{\mathbf{w}}_{k}=\hat{\mathbf{w}}_{k}^{-}+\mathbf{K}_{k}^{\mathbf{w}}\left(\mathbf{z}_{k}-\mathbf{y}_{k}^{-}\right) \\
\mathbf{P}_{\mathbf{w}_{k}}=\left(\mathbf{I}-\mathbf{K}_{k}^{\mathbf{w}} \mathbf{C}_{k}^{\mathbf{w}}\right) \mathbf{P}_{\mathbf{w}_{k}}^{-}
\end{gathered}
$$

where $\mathbf{R}^{\mathbf{x}}$ and $\mathbf{R}^{\mathbf{w}}$ are the observation covariance matrices of the state and parameter filters, respectively. The measured variables represented in vector $\mathbf{z}_{k}$ at each sampling time.

\section{RESULTS}

In this work we have investigated parallel state and parameter estimation with Dual Extended Kalman Filter. The relative accuracies of the filter in case of single parameter $\left(k_{12}\right)$ and dual parameter $\left(k_{12}, k_{e}\right)$ are compared and represented.

The simulations were developed in the MATLAB 2017b software environment.

We have applied 10 days long simulated scenarios with random glucose intake profiles and insulin profiles which have calculated to be able to neutralize the effect of glucose disturbance with respect to the model [10], [14]. In our figure we show only a given representative slice. Due to the length of the simulated time horizons the values cannot be represented within one figure. However, we provide Root Mean Square Errors (RMSE) based assessment as well applied on the whole 10 days long time horizon for verification purposes.

The reference values were generated by solving (1)-(11) using the inbuilt ode45() solver of the MATLAB software. The reference $G(t)$ values were the inputs to the sensor noise model. The output of the system (17)-(21) was used as simulated CGMS data. The inputs of the system follow a manual, arbitrarily chosen carbohydrate and insulin administration scheme. Carbohydrate intakes range between $10[\mathrm{~g}]$ and 120 [g], insulin injections 2 [Unit] and 9 [Unit]. Both of the inputs are bolus like. Previously we referred to carbohydrate intakes as inputs, however from control point of view those are modeled as disturbances. This means the filters have no a priori knowledge about the temporality nor the magnitude of the boluses.

The filters were initialized by adding a minor offset to the reference states and parameters. Important 
to note, that parameters which were not estimated are equal to their reference counterpart. Initial states: $\mathbf{x}_{\text {ref }}(0)=[90,30,0,0.0088,0.0012,0.078,0,0,0,0]^{\top}$ and $\mathbf{x}_{D E K F}(0)=[100,35,0,0.0088,0.0012,0.078,0,0,0,0]^{\top}$. Parameters which are common in case of single and dual parameter estimation are defined as follows: $\lambda=0.99, \mathbf{R}^{\mathbf{x}}=0.2$, $\mathbf{R}^{\mathbf{w}}=0.064$.

\section{A. SINGLE PARAMETER ESTIMATION}

The only measured variable is the blood glucose concentration in the daily life, this means by applying (30)-(42) of the DEKF, only parameters present in the first (1) can be estimated. This is an obvious consequence if we consider the output matrix in (39). In a previous study it was shown that $k_{12}$ - which is the transfer rate from the blood to the tissues [10] - has the most significant effect on the glucose levels from the inputs points of view [10], thus our primary goal was to estimate that parameter beside the state variables.

The process noise covariance matrix was determined by experimental method, keeping in mind the possible variances and uncertainties of each state in accordance with our previous findings [14].

$$
\mathbf{Q}=\left[\begin{array}{ccccccc}
31.5 & 6.25 & \ldots & 125 & 125 & 0 & 0 \\
6.25 & 1.25 & \ldots & 25 & 25 & 0 & 0 \\
\vdots & \vdots & \ddots & \vdots & \vdots & \vdots & \vdots \\
125 & 25 & \ldots & 500 & 500 & 0 & 0 \\
125 & 25 & \ldots & 500 & 500 & 0 & 0 \\
0 & 0 & \ldots & 0 & 0 & 0 & 0 \\
0 & 0 & \ldots & 0 & 0 & 0 & 0
\end{array}\right]
$$

Figure 1 shows the "nominal" $G_{\text {reference }}$ (output of the virtual patient model), the measured $G_{\text {sensor }}=G_{C G M}$ and the filtered $G_{D-D E K F}$ blood glucose levels, respectively. It can be seen that despite the time delay and random Brownian loads the DEKF is able to approach the nominal model with acceptable accuracy. Since $G(t)=Q_{1}(t) / V_{G}$ the diagram can be interpreted to $Q_{1}$ variable which having $k_{12}$ in its equation.

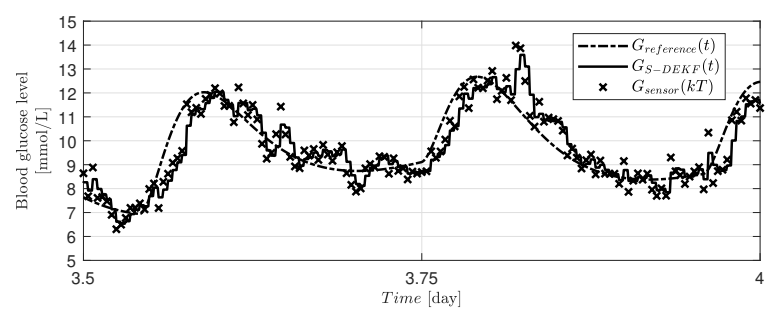

Fig. 1. $G(t)$ output of the DEKF with single parameter estimation.

Figure 2 shows the estimation performance of the DEKF in case of $Q_{2}$. It can be seen the DEKF performed well only producing acceptable error coming from the time delay effect of the noise model.

Figure 3 shows the estimation of the absorption sub-model $D_{1,2}$ in which the highest estimation errors obtained due to

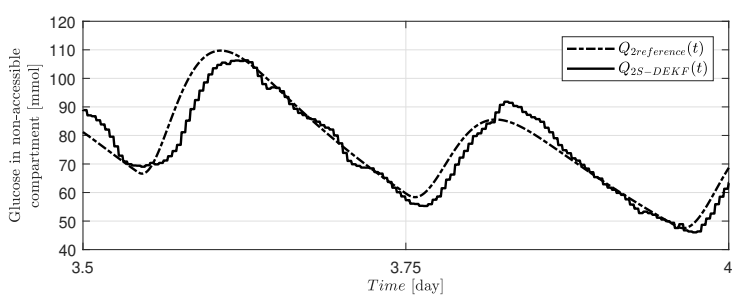

Fig. 2. $Q_{2}(t)$ output of the DEKF with single parameter estimation.

the fact that the DEKF did not have preliminary knowledge about the incoming glucose intakes (disturbances). Nevertheless, the $D_{2}$ state estimation has been acceptable.
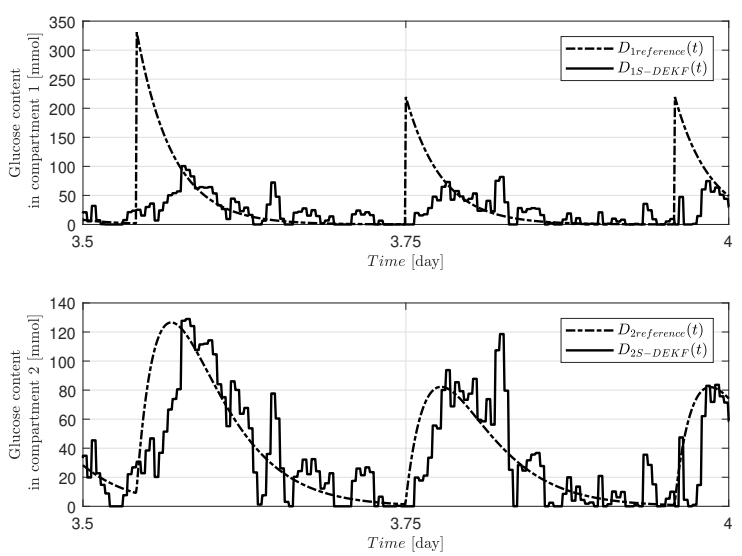

Fig. 3. $D_{1}(t)$ and $D_{2}(t)$ output of the DEKF with single parameter estimation.

Figure 4 shows the $k_{12}$ estimation for the whole simulation horizon. The $k_{12 \text {,reference }}(t)$ is the selected varying patient specific model parameter. Higher estimation error obtained after the initialization due to applied DEKF technique and the time delay effect, however, we found the DEKF can be applied for estimation purposes in this sense. Furthermore, despite the varying estimated $k_{12}$ the accuracy of the state predictions have been acceptable taking into account the applied circumstances.

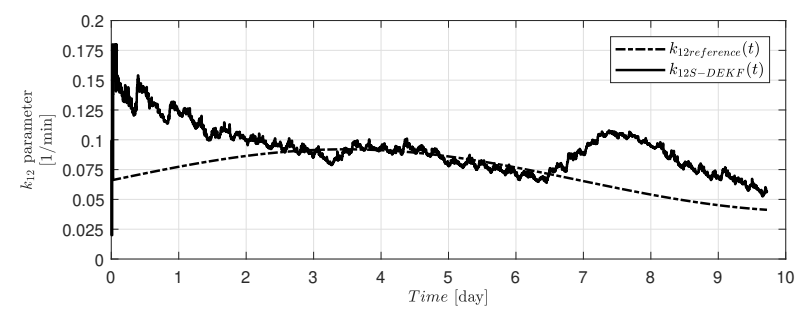

Fig. 4. $k_{12}$ parameter estimation of the DEKF with single parameter estimation. 


\section{B. DUAL PARAMETER ESTIMATION}

Beside $k_{12}, k_{e}$ is also an important parameter of the Cambridge model as it substantially effects the insulin level. Although by strictly interpreting the equations of the applied filter, it is not possible to estimate $k_{e}$. In order to estimate it one has to make certain presumptions about the $I(t)$ state.

Insulin concentration in the blood depends only on the $S_{1}(t), S_{2}(t)$ states and $k_{e}, \tau_{S}, V_{I}$ parameters. Due to $\tau_{S}$ connected to the applied insulin and the $V_{I}$ is related to the fluid space of the patient they can be handled as constant on the investigated time frame. Thus, it can be assumed that $k_{e}$ caused the main deviation of $I(t)$. Thus, the state EKF can be utilized as a parameter estimator in case of $I(t)$. Moreover the relation of the state and the parameter with respect to the derivative of $I(t)$ have to be investigated. It can be seen in (4) that the two variables identically affect the derivative (practically this means e.g. we do not have to change the sign of the estimation during the conversion from state to parameter). Finally the process noise covariance matrix has to be altered. Variances have to be added to the $I(t)$ states, however magnitudes lower compared to the other states because of the small values of the parameter and possible small changes:

$\left[\begin{array}{cccccccc}31.5 & 6.25 & 0.005 & \ldots & 125 & 125 & 0 & 0 \\ 6.25 & 1.25 & 0.003 & \ldots & 25 & 25 & 0 & 0 \\ 0.005 & 0.003 & 8 \cdot 10^{-8} & \ldots & 0.02 & 0.02 & 0 & 0 \\ \vdots & \vdots & \vdots & \ddots & \vdots & \vdots & \vdots & \vdots \\ 125 & 25 & 0.02 & \ldots & 500 & 500 & 0 & 0 \\ 125 & 25 & 0.02 & \ldots & 500 & 500 & 0 & 0 \\ 0 & 0 & 0 & \ldots & 0 & 0 & 0 & 0 \\ 0 & 0 & 0 & \ldots & 0 & 0 & 0 & 0\end{array}\right]$

Figure 5 presents the $G_{\text {reference }}$, the measured $G_{\text {sensor }}=$ $G_{C G M}$ and the filtered $G_{D-D E K F}$ blood glucose levels in case of dual parameter estimation, respectively. Despite the varying $k_{12}$ and $k_{e}$ the signals are quite similar to the previous case with almost the same filtering performance.

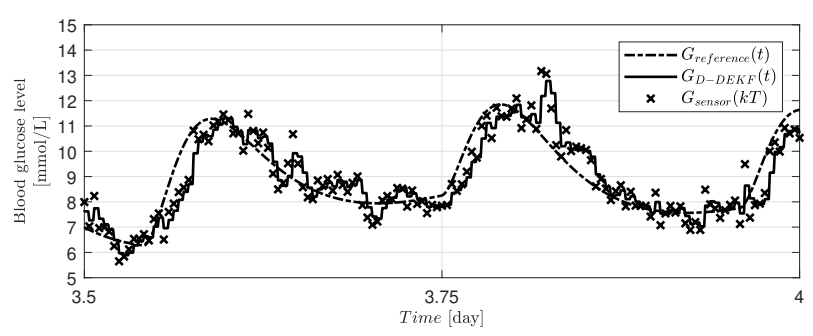

Fig. 5. $G(t)$ output of the DEKF with dual parameter estimation.

Figure 6 shows the $Q_{2}$ state variables and estimation. It can be seen that the estimation performance is similar as in the previous case.

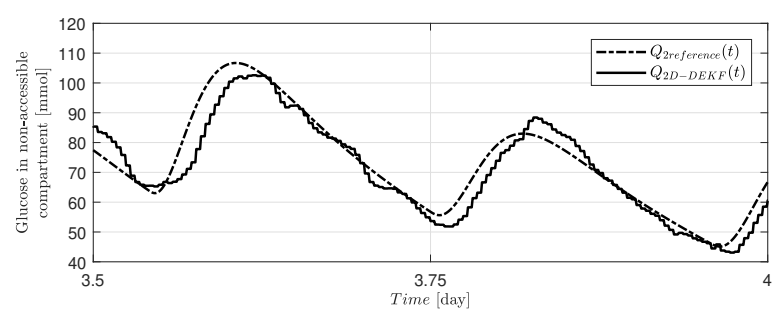

Fig. 6. $Q_{2}(t)$ output of the DEKF with dual parameter estimation.

Figure 8 shows the $I$ state variables and estimation. Despite the varying $k_{e}$ parameter the DEKF is able to estimate the state with high accuracy.

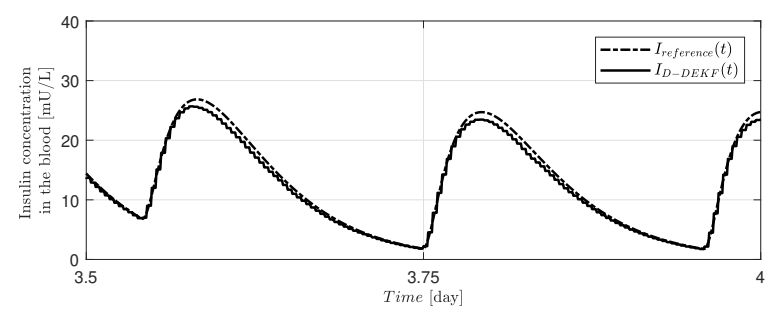

Fig. 7. $I(t)$ output of the DEKF with dual parameter estimation.

Figure 8 shows the estimation of $D_{1,2}$. It can be seen that the DEKF's performance was similar.
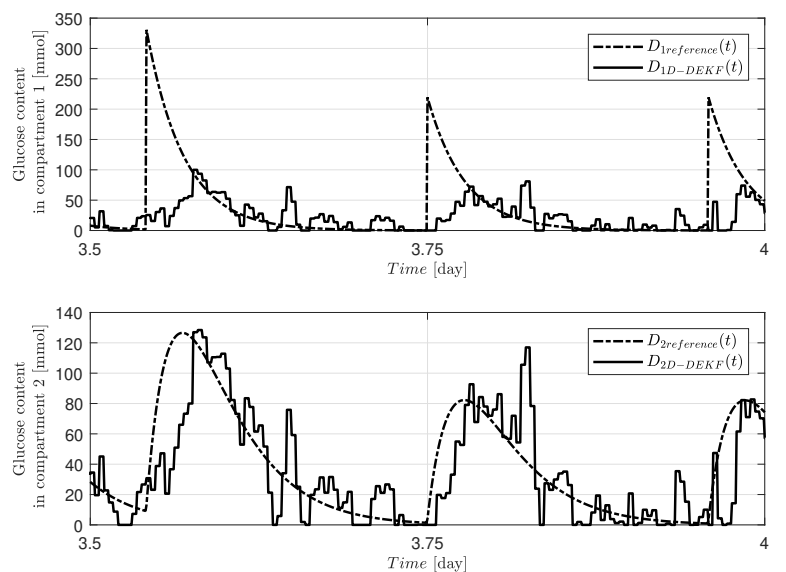

Fig. 8. $D_{1}(t)$ and $D_{2}(t)$ output of the DEKF with dual parameter estimation.

The most important diagram is Fig. 9 in which both the changes and estimations of $k_{12}$ and $k_{e}$ can be found. In both cases the estimation accuracy was around $75 \%$ - which is acceptable compared to the applied framework and varying signals. 

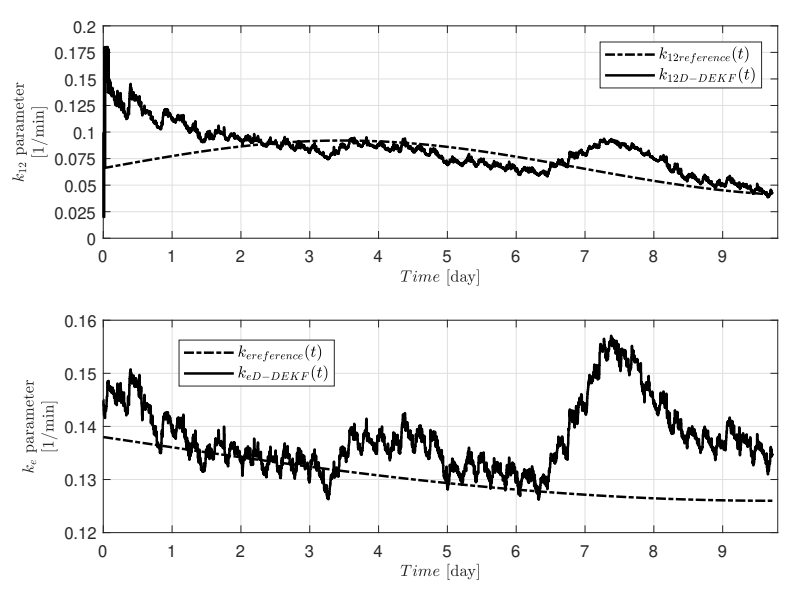

Fig. 9. $k_{12}$ and $k_{e}$ parameter estimation of the DEKF.

\section{NUMERICAL ASSESSMENT}

In order to better grasp the performance of the filter in case of single parameter and dual parameter estimation a numerical assessment was made. The states and parameters are first normalized to $[0,1]$, between the normalized values RMSE have calculated: $\left\|\mathbf{x}_{n-\text { reference }}(t)-\mathbf{x}_{n-D E K F}(k T)\right\|$ in Table I. The estimation of two parameters (D-DEKF) has only minor effect on the states which already had significant uncertainty $\left(Q_{1}(t), Q_{2}(t), D_{1}(t), D_{2}(t)\right)$. The error in the remaining states in case of the single parameter estimation (S-DEKF) is solely caused by the discretization procedure, zero-order hold and numerical calculations. The realization of the estimation of the second parameter $\left(k_{e}\right)$ introduces further differences in the states which are directly or indirectly dependent on $k_{e}$. The increased error in $I(t)$, $x_{1}(t), x_{2}(t), x_{3}(t)$ is caused by the deviation of $k_{e}$ from its reference value.

TABLE I

RMSE ERRORS

\begin{tabular}{lllllll}
\hline Filters & $Q_{1}(t)$ & $Q_{2}(t)$ & $I(t)$ & $x_{1}(t)$ & $x_{2}(t)$ & $x_{3}(t)$ \\
\hline S-DEKF & 7.34 & 14.23 & 0.780 & 0.354 & 0.681 & 0.607 \\
D-DEKF & 7.38 & 15.7 & 2.44 & 5.86 & 2.44 & 2.56 \\
\hline & & & & & & \\
\hline Filters & $D_{1}(t)$ & $D_{2}(t)$ & $S_{1}(t)$ & $S_{2}(t)$ & $k_{12}(t)$ & $k_{e}(t)$ \\
\hline S-DEKF & 21.8 & 14.3 & 1.24 & 0.880 & 42.8 & - \\
D-DEKF & 22.0 & 14.9 & 1.24 & 0.880 & 45.0 & 44.7 \\
\hline
\end{tabular}

\section{CONCLUSIONS}

In this paper we have introduced the development of a DEKF framework which is able to estimate both the state variables and model parameters simultaneously by utilizing the discrete LPV methodology.

We have developed given qLPV model based on the nonlinear Cambridge T1DM model and we applied a nonlinear noise model to map the noise effects appear during the application of CGM system.
The graphical and numerical results have shown that the performance of the developed DEKF is acceptable and we can use it in our future work related to controller development. The main estimation errors obtained due to the time delay and unknown glucose disturbance.

In our future work we will improve on the DEKF algorithm and adding extra smoothers in order to decrease the error on the states and parameters.

\section{REFERENCES}

[1] International Diabetes Federation, IDF Diabetes Atlas, 8th ed. Brussel, Belgium: International Diabetes Federation, 2017.

[2] R. DeFronzo, E. Ferrannini, P. Zimmet, and G. Alberti, International Textbook of Diabetes Mellitus, 2 Volume Set, 4th ed. Wiley-Blackwell, 2015.

[3] T. Danne, R. Nimri, T. Battelino, R. M. Bergenstal, K. L. Close, J. H. DeVries, S. Garg, L. Heinemann, I. Hirsch, S. A. Amiel et al., "International consensus on use of continuous glucose monitoring," Diabetes care, vol. 40, no. 12, pp. 1631-1640, 2017.

[4] A. Facchinetti, S. Del Favero, G. Sparacino, J. R. Castle, W. K. Ward, and C. Cobelli, "Modeling the glucose sensor error," IEEE T Biomed Eng, vol. 61, no. 3, pp. 620-629, 2014.

[5] L. M. Huyett, E. Dassau, H. C. Zisser, and F. J. Doyle III, "Glucose Sensor Dynamics and the Artificial Pancreas: The Impact of Lag on Sensor Measurement and Controller Performance," IEEE Contr Syst, vol. 38 , no. 1 , pp. 30-46, 2018.

[6] R. Nimri, A. Murray, N. abd Ochs, J. Pinsker, and E. Dassau, "Closing the Loop," Diabetes Technol The, vol. 19, no. 1, pp. s27 - s41, 2017.

[7] M. Grewal and A. Andrews, Kalman Filtering: Theory and Practice Using MATLAB, 4th ed. Chichester, UK: John Wiley \& Sons, 2014.

[8] D. Boiroux, M. Hagdrup, Z. Mahmoudi, K. Poulsen, H. Madsen, and J. B. Jørgensen, "An ensemble nonlinear model predictive control algorithm in an artificial pancreas for people with type 1 diabetes," in 2016 European Control Conference (ECC). IEEE, 2016, pp. 21152120 .

[9] R. Hovorka, V. Canonico, L. Chassin, U. Haueter, M. Massi-Benedetti, M. Orsini-Federici, T. Pieber, H. Schaller, L. Schaupp, T. Vering, and W. M.E., "Nonlinear model predictive control of glucose concentration in subjects with type 1 diabetes," Physiol Meas, vol. 25, no. 4, pp $905-920,2004$

[10] M. Naerum, "Model predictive control for insulin administration in people with type 1 diabetes," Technical University of Denmark, Tech. Rep., 2010.

[11] D. Boiroux, V. Bátora, M. Hagdrup, S. L. Wendt, N. K. Poulsen, H. Madsen, and J. B. Jørgensen, "Adaptive model predictive control for a dual-hormone artificial pancreas," Journal of Process Control, vol. 68 , pp. $105-117,2018$.

[12] G. Eigner, "Control of physiological systems through linear parameter varying framework," Acta Polytechnica Hungarica, vol. 14, no. 6, pp. 185-212, 2017.

[13] A. White, G. Zhu, and J. Choi, Linear Parameter Varying Control for Engineering Applicaitons, 1st ed. London: Springer, 2013.

[14] G. Eigner, M. Siket, and L. Kovács, "Kalman filtering of discrete lpv diabetes mellitus model for control purposes," in IEEE 18th International Symposium on Computational Intelligence and Informatics. IEEE, 2018, pp. 119-124.

[15] R. Tóth, P. S. Heuberger, and P. M. Van den Hof, "Discretisation of linear parameter-varying state-space representations," IET control theory \& applications, vol. 4, no. 10, pp. 2082-2096, 2010.

[16] R. Tóth, Modeling and identification of linear parameter-varying systems. Springer, 2010, vol. 403.

[17] E. A. Wan and A. T. Nelson, "Dual extended kalman filter methods," Kalman filtering and neural networks, vol. 123, 2001.

[18] T. A. Wenzel, K. Burnham, M. Blundell, and R. Williams, "Dual extended kalman filter for vehicle state and parameter estimation," Vehicle System Dynamics, vol. 44, no. 2, pp. 153-171, 2006.

[19] A. Popovici, P. Zaal, and D. M. Pool, "Dual extended kalman filter for the identification of time-varying human manual control behavior,' in AIAA Modeling and Simulation Technologies Conference, 2017, p. 3666 